\title{
Probing Galactic cosmic rays with $\gamma$-ray observations of giant molecular clouds
}

\section{Giada Peron, ${ }^{a, *}$ Felix Aharonian, ${ }^{a, b}$ Sabrina Casanova, ${ }^{a, c}$ Ruizhi Yang ${ }^{d, e}$ and Roberta $\operatorname{Zanin}^{f}$}

${ }^{a}$ Max-Planck-Institut für Kernphysik, Saupfercheckweg 1, Heidelberg, Germany

${ }^{b}$ Dublin Institute for Advanced Studies, 31 Fitzwilliam Place, Dublin 2, Ireland

${ }^{c}$ Institute of Nuclear Physics PAN,

Radzikowskiego 152, 31-342 Kraków, Poland

${ }^{d}$ CAS Key Laboratory for Research in Galaxies and Cosmology, Department of Astronomy, University of Science and Technology of China,

Hefei, Anhui 230026, China

${ }^{e}$ School of Astronomy and Space Science, University of Science and Technology of China, Hefei, Anhui 230026, China

${ }^{f}$ CTA observatory, Via Piero Gobetti 93/3 40129 Bologna, Italy

E-mail: giada.peron@mpi-hd.mpg.de

High-energy $\gamma$ rays originating from interactions of cosmic rays (CRs) with the interstellar medium (ISM) carry direct information about the spatial and spectral distribution of these relativistic particles. Fermi-LAT observations of the diffuse gas emission show enhanced emission in the region around $4 \mathrm{kpc}$ from the Galactic center. Analyses of the diffuse emission however are performed on a large spatial scale, usually of several $\mathrm{kpc}^{2}$. Giant Molecular clouds instead are a unique tool, which can be used as 'barometers' to infer the cosmic-ray density point by point, in distant and small regions of the Galaxy. Their enhanced density $\left(\mathrm{n}_{H}>100 \mathrm{~cm}^{-} 3\right)$, compared to the diffuse gas, allows us to derive the CR energy density on scales comparable to the size of the clouds (10-100 pc). We report here the results of the analyses of Fermi-LAT Pass 8 data, obtained in the direction of molecular clouds located in the entire galactic disk from $0.1 \mathrm{kpc}$ to $12 \mathrm{kpc}$ from the Galactic Center (GC). The CR densities measured at the locations of these clouds have a high degree of fluctuation and are not always compatible with the values derived from the diffuse gas.

$37^{\text {th }}$ International Cosmic Ray Conference (ICRC 2021)

July 12th - 23rd, 2021

Online - Berlin, Germany

\footnotetext{
*Presenter
} 


\section{Introduction}

The theory of acceleration and propagation of Galactic cosmic rays (CRs) is built on direct observations of these high energetic particles in the vicinity of the Earth [6]. The local CR spectrum has a steep power-law shape of average index $\Gamma \sim 2.7$, up to the so-called knee at a few PeV, which is considered to be the edge of CRs of Galactic origin. The shape of the spectrum is believed to be the result of the modulation $\delta$ of the injected spectrum, $E^{-\alpha}$, caused by the diffusive propagation, which CR undergo in the Galaxy: $\Gamma=\alpha+\delta$. Moreover, we know that CRs of $<1 \mathrm{PeV}$ energies are confined for at least $10^{7}$ years so we expect them to spread almost uniformly in the entire Galaxy, forming the so-called CR "sea". Testing whether there is a uniform sea and if its level coincide with the value of cosmic ray density detected locally, is of fundamental importance to validate the theories. In order to test this hypothesis, one should probe the CR spectrum in locations far from the Earth. While direct CR observations cannot tell about the origin of the particle, $\gamma$-ray emission originating by the interaction of CRs with the interstellar medium (ISM) directly trace the distribution of cosmic rays at the location of the interaction and therefore are a unique channel to explore CRs far from the Earth.

Several analyses were dedicated to study the $\gamma$-ray diffuse emission unveiling a inhomogeneous $\mathrm{CR}$ density, peaked in a region around $4 \mathrm{kpc}$ from the Galactic center, where the spectrum is also slightly harder than the local one $[1,12,17]$. This can be due to different phenomena: a higher density of CR accelerator in the inner Galaxy, the erroneous inclusion of unresolved sources in the emission or the spatial dependency of the CR diffusion coefficient. Measurements obtained from the diffuse emission however are conducted on a very large scale, usually of several $\mathrm{kpc}^{2}$ and therefore report an average value, which can be biased by the presence of single regions with enhanced and harder flux. When targeting single molecular clouds (MCs) instead,not only we are able to localize the cosmic rays, but we are also less concerned by contamination of unresolved or mis-modeled sources. Moreover, as [8] points out, most of the line of sights are dominated by just a few $(<3)$ clouds, therefore the values of the diffuse emission could be wrongly interpreted to concern a larger area.

\section{Molecular clouds selection}

The interstellar medium (ISM) of the Milky way can be traced either by collision-excited molecular lines (e.g. ${ }^{12} \mathrm{CO}(1 \rightarrow 0)$; e.g. [5]), by spin-flip transition lines (HI $21 \mathrm{~cm} \mathrm{[4])} \mathrm{or} \mathrm{by} \mathrm{dust}$ opacity maps [2]. Analyzing the all-sky CO survey of [5], several catalogs of molecular cloud were produced. The most recent are the one by Rice et al. [13] and the one of Miville Deschenes et al. [8], which contain 1063 and 8107 molecular clouds respectively. Previously, detection of $\gamma$-rays from molecular clouds were limited to very close clouds (e.g. Gould belt clouds; $[10,16]$ ) or to the extremely massive MC complex at the galactic center [15]. The new catalogs containing several objects with masses overcoming $10^{6} \mathrm{M}_{\odot}$, opened up new possibilities to extract the CR density also at intermediate densities.

The gamma-ray flux expected from a molecular cloud illuminated by penetrating cosmic rays is proportional to the cloud column density, which can be express in terms of $A=M_{5} / d_{k p c}^{2}$, where $M_{5}=10^{5} \mathrm{M}_{\odot}$ and $d_{k p c}$ is the distance in kpc: 


$$
F_{\gamma} \propto A \phi_{\gamma}(E) \propto A \xi_{N} \int d E \frac{d \sigma}{d E} \frac{d N_{p}}{d E d A d t d \Omega}
$$

where $\xi_{N}$ is the nuclear enhancement factor, which is $\xi_{N}=1.8$ for the local composition of ISM and CRs at $10 \mathrm{GeV}$ [9] and $d \sigma / d E$ is the proton-proton cross section [7]. It is clear, that by measuring $F_{\gamma}$, and knowing the cloud column density, one can derive the proton spectrum $\frac{d N_{p}}{d E d A d t d \Omega}$.

As explained in [3], $A=0.4$ is a good threshold to allow a spectral detection of molecular clouds with Fermi-LAT both in the inner and in the outer Galaxy. However, only $~ 3 \%$ of clouds in the Miville-Deschênes catalog overcomes this threshold.

To guarantee the quality of the detection, we minimized source confusion by discarding all the clouds which had a overlapping known gamma-ray source. In a second step, we explored the velocity distribution of the gas and we chose those clouds which dominated the gas density along the line of sight. All the chosen clouds occupies at least $40 \%$ of their column. The remaining fraction of gas along the line of sight has to be modeled independently in the analysis in order to guarantee a detection at the location of the cloud. This can be done by separating the 3 dimensional distribution $(\Delta l, \Delta b, \Delta v)$ of the cloud from the rest of the gas, as explained in the following section. Maps based on line emission like $\mathrm{CO}$ and HI, contain the information on the gas radial velocity and therefore on the gas distribution along the line of sight, while dust opacity maps are bi-dimensional. For these reasons, in principle, one should use dust only when the line of sight is totally dominated by the cloud of interest, like in the case of Gould Belt clouds. However, CO+HI template although advantageous to study MCs at low latitudes suffer of large uncertainties, related to saturation and self absorption. Moreover, in certain region of the inner Galaxy, the velocity distribution is not well determined, impeding a proper separation of the gas components on the line of sight [11]. In this cases, it is convenient to look at the entire line of sight and, even if it is not be possible to localize the origin of the emission, one can put constraining upper limits to the CR density and on its deviation from the local value. In particular, this method is successful to determine an enhancements, $N$, above the nominal level of a certain fraction of gas, $f_{c}$, if the enhancement is at least:

$$
N>1.85 \frac{f_{c}}{1-f_{c}}
$$

In order to calculate $f_{c}$, one can make some reasoning on the MC distribution, [11], or whether possible use other optically thin tracers.

Our final sample was composed by 28 molecular clouds distributed in different part of the Galaxy located from $0.5 \mathrm{kpc}$ to $12 \mathrm{kpc}$. That included 3 clouds of the Gould Belt complex and 3 other nearby ( $\lesssim 2 \mathrm{kpc}$ ) high latitude molecular clouds, namely Cepheus, Monoceros OB2 and Maddalena. 12 molecular clouds from the Rice et al. catalog belonging to the Galactic plane at intermediate distances, 9 molecular clouds of the Miville Deschenes catalog located within 1.5 and $4.5 \mathrm{kpc}$, the region where the Fermi-LAT collaboration report an enhanced gamma-ray emissivity. Finally, the Sgr B complex, at the very center of the Galaxy ( $\sim 0.1 \mathrm{kpc})$ was re-analyzed following the procedure of [15]. 

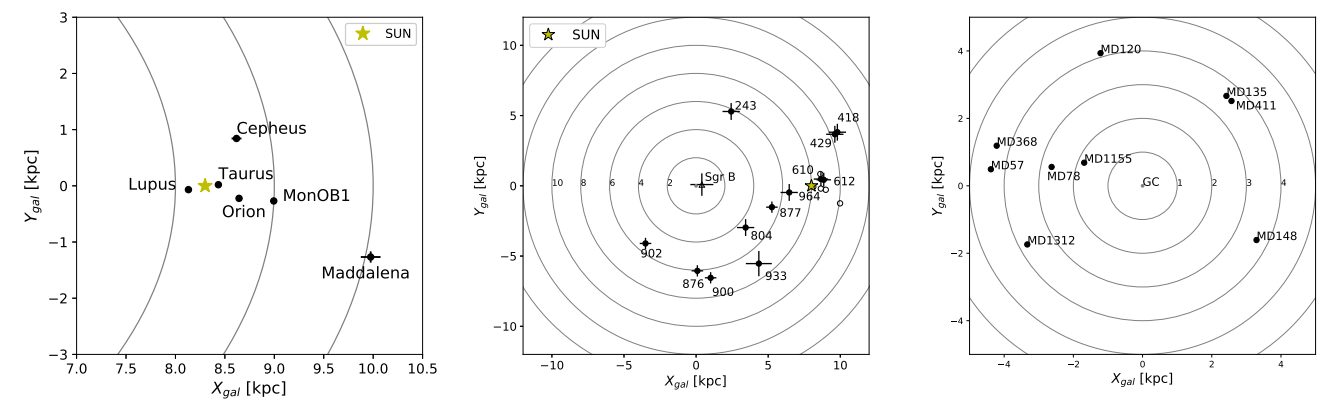

Figure 1: Distribution of the analyzed clouds in the Galaxy.

\section{Observations}

We used fermipy to analyze Pass8 Fermi-LAT data in the region corresponding to the selected clouds. Since results from different analysis are presented here, we refer to the main publications for the details on the data selection and quality cuts $[3,11]$. In the standard Fermi-LAT analysis routine a unique model for the galactic diffuse emission is employed, that includes the emission from pion decay, inverse Compton, and bremsstrahlung in a unique template. To extract the spectrum of the clouds instead one needs to create a separate model for it. To account for all different components, our model included a template for inverse Compton emission, generated by galprop [14], a spatial template for the cloud (based either on the CO [5], or dust opacity [2], depending on the position of the cloud) and a template for the remaining diffuse emission. This allows us to account for all the emission components, while treating the region of the cloud as an independent component.

After a first fit of the large scale templates, we proceeded by optimizing the known point-like sources from the 4th Fermi LAT catalog (4FGL). We then modeled all the significant (TS $>25$ ) residual emission and then extracted the spectral energy distribution (SED) by fitting the normalization in each energy bin.

From the resulting SEDs, we extracted the parameters $\left(\rho_{C R}\right.$ and $\left.\alpha_{C R}\right)$ of the parent CR protons, with the use of naima. The extracted parameters are reported in figure 2 as a function of the cloud galactocentric distance. The results show different behavior according to the region of the Galaxy where the cloud belongs to. As one can see, in the outer regions, the clouds show a steep spectrum, with a normalization comparable to the local CR spectrum. Going to inner regions instead, we see some deviations with clouds showing harder and higher spectra. When enhanced, the values are not always consistent with a unique value and are not compatible with the values reported by the analysis of the diffuse rings. This is particularly evident for the region between $1.5 \mathrm{kpc}$ and $4.5 \mathrm{kpc}$, where the flux extracted from the clouds, is significantly lower than the ring average.

\section{Conclusions and outlook}

The results that we obtained from the studied clouds allow us to drive several conclusions. First of all, the identification of several different regions with similar CR spectra as observed at Earth clearly proves that we do not live in a special place, but that similar level of CR density can be achieved also in other part of the Milky Way. Differences are found only in the inner Galaxy, 

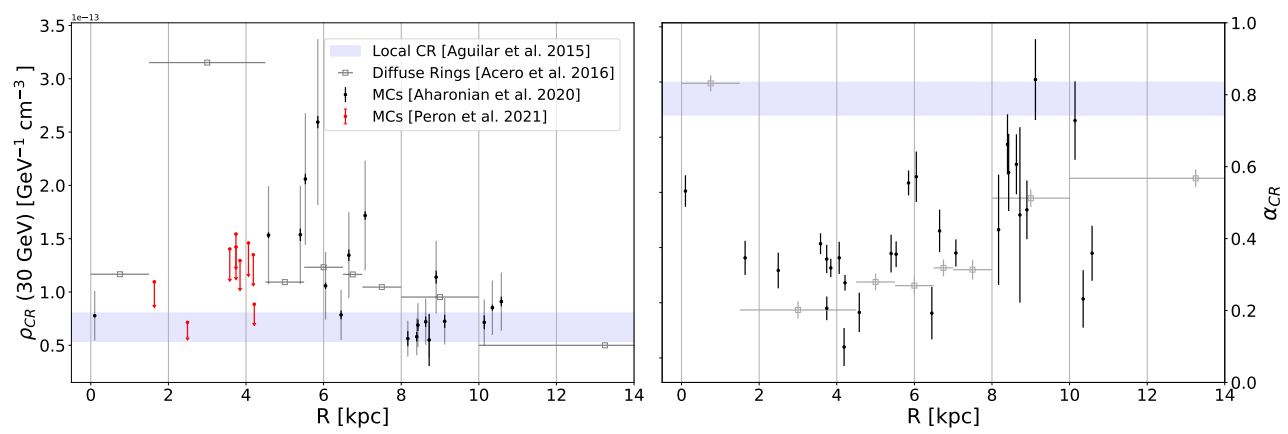

Figure 2: The parameters (density at $30 \mathrm{GeV}$ and spectral index) of cosmic rays, derived from the analyzed molecular clouds as a function of the galactocentric distance of the cloud. The light errorbar indicates the systematic uncertainty. The points are compared with the values derived from the analysis of the diffuse (grey squares). The blue area corresponds to a proton flux that coincides with the one measured by AMS02, the extent of the area accounts for the $20 \%$ systematic uncertainty that derives from the gas column density.

where we expect a higher density of CR accelerators and therefore deviations possibly arises by the higher density of freshly accelerated particles. An hint of this claim comes from the large fluctuations of the parameters extracted from clouds at similar distances $(\sim 6 \mathrm{kpc})$, which point towards a random variation as generated by source vicinity, rather than a global variation of the CR diffusion properties. Lastly, the points derived in the inner $(1.5-4.5 \mathrm{kpc})$ ring, show a huge discrepancy with the emissivity calculated for the ring. This is an indication that the analysis of the large scale diffuse emission is not a good indication of the average value as it is biased by the presence of a few enhanced clouds and it is not sensitive to local variations.

In order to probe the sea of cosmic rays ideally we need to extend these observations to as many as possible regions. Unfortunately the number of dense enough cloud to produce detectable emission is limited in the Galaxy. If the sensitivity of Fermi-LAT would increase of at least a factor of 2, a much wider area of the Galaxy could be probed, see 3. Such an improvement of the sensitivity hardly can be obtained by increasing the exposure of the LAT, but it could be achieved by a similar instrument with a larger area.

\section{References}

[1] F. Acero, M. Ackermann, M. Ajello, A. Albert, L. Baldini, J. Ballet, G. Barbiellini, D. Bastieri, R. Bellazzini, E. Bissaldi, E. D. Bloom, R. Bonino, E. Bottacini, T. J. Brandt, J. Bregeon, P. Bruel, R. Buehler, S. Buson, G. A. Caliandro, R. A. Cameron, M. Caragiulo, P. A. Caraveo, J. M. Casandjian, E. Cavazzuti, C. Cecchi, E. Charles, A. Chekhtman, J. Chiang, G. Chiaro, S. Ciprini, R. Claus, J. Cohen-Tanugi, J. Conrad, A. Cuoco, S. Cutini, F. D’Ammando, A. de Angelis, F. de Palma, R. Desiante, S. W. Digel, L. Di Venere, P. S. Drell, C. Favuzzi, S. J. Fegan, E. C. Ferrara, W. B. Focke, A. Franckowiak, S. Funk, P. Fusco, F. Gargano, D. Gasparrini, N. Giglietto, F. Giordano, M. Giroletti, T. Glanzman, G. Godfrey, I. A. Grenier, S. Guiriec, D. Hadasch, A. K. Harding, K. Hayashi, E. Hays, J. W. Hewitt, A. B. Hill, D. Horan, X. Hou, T. Jogler, G. Jóhannesson, T. Kamae, M. Kuss, D. Landriu, S. Larsson, 


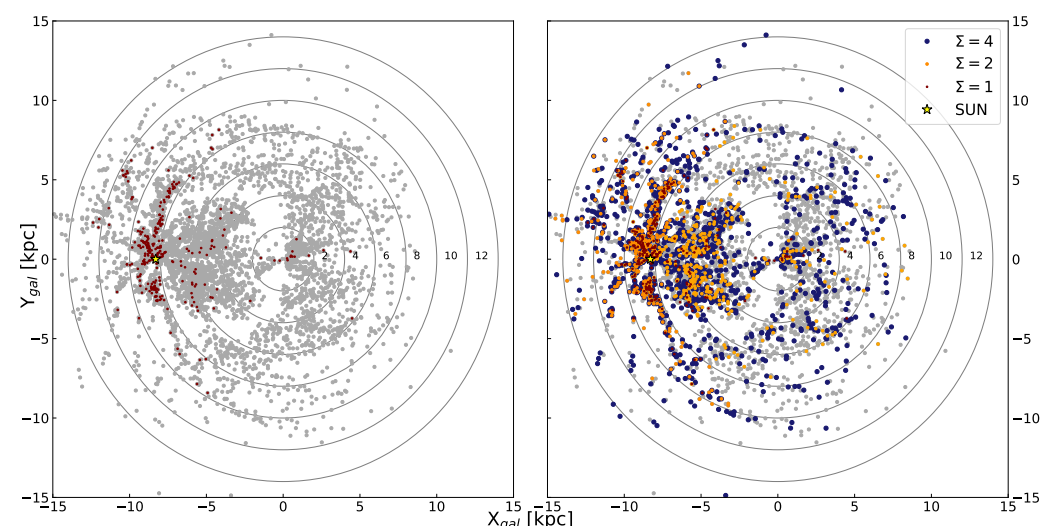

Figure 3: Position on the galactic plane of the molecular clouds of [8]. The grey points show clouds which are not visible due to their low A factor. Dark red points are clouds detectable with the current Fermi-LAT 10 years sensitivity, orange ones and blue ones are clouds that would be detectable if the sensitivity of Fermi-LAT would be improved of a factor 2 or 4 respectively.

L. Latronico, J. Li, L. Li, F. Longo, F. Loparco, M. N. Lovellette, P. Lubrano, S. Maldera, D. Malyshev, A. Manfreda, P. Martin, M. Mayer, M. N. Mazziotta, J. E. McEnery, P. F. Michelson, N. Mirabal, T. Mizuno, M. E. Monzani, A. Morselli, E. Nuss, T. Ohsugi, N. Omodei, M. Orienti, E. Orlando, J. F. Ormes, D. Paneque, M. Pesce-Rollins, F. Piron, G. Pivato, S. Rainò, R. Rando, M. Razzano, S. Razzaque, A. Reimer, O. Reimer, Q. Remy, N. Renault, M. Sánchez-Conde, M. Schaal, A. Schulz, C. Sgrò, E. J. Siskind, F. Spada, G. Spandre, P. Spinelli, A. W. Strong, D. J. Suson, H. Tajima, H. Takahashi, J. B. Thayer, D. J. Thompson, L. Tibaldo, M. Tinivella, D. F. Torres, G. Tosti, E. Troja, G. Vianello, M. Werner, K. S. Wood, M. Wood, G. Zaharijas, and S. Zimmer. Development of the Model of Galactic Interstellar Emission for Standard Point-Source Analysis of Fermi Large Area Telescope Data. The Astrophysical Journal Supplement Series, 223(2), feb 2016.

[2] P.A.R Ade, N. Aghanim, M. Arnaud, and M. Ashdown. Planck early results. XIX. All-sky temperature and dust optical depth from Planck and IRAS. Constraints on the "dark gas" in our Galaxy. Astronomy \& Astrophysics, 536:16, 2011.

[3] Felix Aharonian, Giada Peron, Ruizhi Yang, Sabrina Casanova, and Roberta Zanin. Probing the sea of galactic cosmic rays with Fermi-LAT. Physical Review D, 101(8), 2020.

[4] N. Ben Bekhti, L. Flöer, R. Keller, J. Kerp, D. Lenz, B. Winkel, J. Bailin, M. R. Calabretta, L. Dedes, H. A. Ford, B. K. Gibson, U. Haud, S. Janowiecki, P. M.W. Kalberla, F. J. Lockman, N. M. McClure-Griffiths, T. Murphy, H. Nakanishi, D. J. Pisano, and L. Staveley-Smith. HI4PI: A full-sky Hi survey based on EBHIS and GASS. Astronomy and Astrophysics, 594, oct 2016.

[5] T M Dame, Dap Hartmann, and P Thaddeus. The Milky Way in Molecular Clouds: A New Complete CO Survey. Technical report. 
[6] Stefano Gabici, Carmelo Evoli, Daniele Gaggero, Paolo Lipari, Philipp Mertsch, Elena Orlando, Andrew Strong, and Andrea Vittino. The origin of Galactic cosmic rays: challenges to the standard paradigm. International Journal of Modern Physics D, 28(15), mar 2019.

[7] Ervin Kafexhiu, Felix Aharonian, Andrew M Taylor, and Gabriela S Vila. Parametrization of gamma-ray production cross-sections for pp interactions in a broad proton energy range from the kinematic threshold to PeV energies. Physical Review D, 90(12):123014, 2014.

[8] Marc-Antoine Miville-Deschênes, Norman Murray, and Eve J. Lee. Physical properties of molecular clouds for the entire Milky Way disk. The Astrophysical Journal, 834(1):57, oct 2016.

[9] Masaki Mori. Nuclear enhancement factor in calculation of Galactic diffuse gamma-rays: A new estimate with DPMJET-3. Astroparticle Physics, 31(5):341-343, jun 2009.

[10] Andrii Neronov, Denys Malyshev, and Dmitri V. Semikoz. Cosmic-ray spectrum in the local Galaxy. Astronomy and Astrophysics, 606, oct 2017.

[11] G. Peron, F. Aharonian, S. Casanova, R. Yang, and R. Zanin. Probing the cosmic-ray density in the inner galaxy. Astrophysical Journal Letters, 907(1), 2021.

[12] Mart Pothast, Daniele Gaggero, Emma Storm, and Christoph Weniger. On the progressive hardening of the cosmic-ray proton spectrum in the inner Galaxy. Journal of Cosmology and Astroparticle Physics, 2018(10):45, 2018.

[13] Thomas S Rice, Alyssa A Goodman, Edwin A Bergin, Christopher Beaumont, and T M Dame. A Uniform Catalog of Molecular Clouds in the Milky Way. The Astrophysical Journal, 822, 2016.

[14] A. E. Vladimirov, S. W. Digel, G. Jóhannesson, P. F. Michelson, I. V. Moskalenko, P. L. Nolan, E. Orlando, T. A. Porter, and A. W. Strong. GALPROP WebRun: An internet-based service for calculating galactic cosmic ray propagation and associated photon emissions. Computer Physics Communications, 182(5):1156-1161, may 2011.

[15] Rui-Zhi Yang, David I Jones, and Felix Aharonian. Fermi-LAT observations of the Sagittarius B complex. Astronomy and Astrophysics, 580:90, 2015.

[16] Rui-zhi Yang, Emma de Oña Wilhelmi, and Felix Aharonian. Probing Cosmic Rays in Nearby Giant Molecular Clouds with the Fermi Large Area Telescope. Astronomy and Astrophysics, 566:142, mar 2013.

[17] Ruizhi Yang, Felix Aharonian, and Carmelo Evoli. Radial distribution of the diffuse $\gamma$-ray emissivity in the Galactic disk. Physical Review D, 93(12), jun 2016. 